\title{
Syncope: dietary advice to help manage the symptoms of syncope \\ Wright $\mathrm{CI}^{*}$ and Thakore $\mathrm{E}$
}

Red Pharm; Cold Meece Estate, Cold Meece, Staffordshire, ST15 0SP, UK

\begin{abstract}
Syncope is defined as a temporary, rapid loss of consciousness caused by fall in blood pressure that is relatively short-lasting. On a population level, it is estimated to affect around 1 in 3 people. The causes of syncope are many with common examples including fainting at the sight of blood or passing-out whilst coughing. The three most common causes of syncope include reduced blood pressure (commonly referred to as orthostatic hypotension), reflex induced changes in blood pressure in response to a stimulus such as the sight of needles or blood and lastly an underlying cardiovascular disease or condition. The aim of this article is to outline the three main causes of syncope as well as outline diets, both in terms of food and fluid intake, can be used to manage the symptoms of syncope.
\end{abstract}

\section{Introduction}

The medical term for fainting is 'syncope' derived from Greek word 'synkope' meaning contraction or cutting off. Around $30 \%$ of people experience a loss of consciousness due to a syncope event during their lifetime; however, its cause remains unclear [1]. Fainting is characterised as the "...transient loss of consciousness caused by insufficient blood flow to the brain characterized by a rapid onset, short duration, and spontaneous and complete recovery" [1]. Syncope is a condition that seems unique to humans [2], although the search for acute models of syncope continues [3]. The most common causes of syncope are depicted in Figure 1 and are discussed in more detail below.

\section{Orthostatic hypotension and syncope}

Orthostatic hypotension is commonly referred to as low blood pressure and can occur acutely when going from a sitting to standing position. When standing-up blood pressure momentarily drops and heart rate increases to counteract the pressure change. This typically occurs when relaxing in a warm bath for a long period and then suddenly standing upright. Orthostatic hypotension can occur as a side effect to medical treatments and drinking alcohol can also lead to a fall in blood pressure and increase the prevalence of syncope. The symptoms typically experienced include sweating, nausea, lightheadedness and visual blurring [1].

\section{Reflex and syncope}

A reflex has a stimulus (input) and leads to a reaction (output) and in this scenario syncope is the body's natural response to a stimulus such as prolonged standing. One common example is seen in soldiers that stand to attention whilst on parade for hours on end [4]. The stimulus in this case is the stationary position which leads to blood pooling in the legs and effectively reducing the volume of blood circulating through the cardiovascular system. Heat works to exacerbate this process as blood pools in dilated vessels and this triggers the fainting episode [5]. The question is what the trigger for the dilatation of vessels? One of the classical triggers for syncope was the stimulation of left ventricular mechanoreceptors [6]. Oberg and Thoren reported that stimulation of left ventricular mechanoreceptors lead to a marked reduction in heart rate and reflex dilatation of arterial resistance and venous capacitance vessels [6]. A mechanism later disproved by Wright et al. [7] in which independent stimulation of left ventricular mechanoreceptors led to minimal changes in heart rate and arterial resistance.

\section{Cardiovascular origins and syncope}

The last cause relates to abnormalities arising from the heart. This type of syncope is usually the result of abnormalities often seen abnormally fast or slow heart rates, and in more severe cases even sudden death [1]. Indeed, Fabrice Muamba, a professional footballer that played for Bolton Wanderers, collapsed during a football game against Tottenham Hotspurs in 2012 [8]. This was linked to hypertrophic obstructive cardiomyopathy, which is more commonly referred to 'HOCM' and defined by the American Heart Association [9] as: "Hypertrophic cardiomyopathy occurs if heart muscle cells enlarge and cause the walls of the ventricles (usually the left ventricle) to thicken. The ventricle size often remains normal, but the thickening may block blood flow out of the ventricle. If this happens, the condition is called obstructive hypertrophic cardiomyopathy". A recent study [10] investigated the relation of syncope and sudden death in 1,511 patients with HOCUM. Authors reported a five times higher rate of sudden death in patients with unexplained syncope, than patients without syncope, and that this was even higher if the patient had experienced a syncope event within 6 months of their initial HOCUM evaluation.

It is important to also mention that other causes of syncope include heart disease per se, a hyperactive thyroid gland and certain cardiovascular medicines as they can lead to syncope in some patients.

Correspondence to: Christopher Ian Wright, Red Pharm, Cold Meece Estate, Cold Meece, Staffordshire, ST15 0SP, UK, Tel: 4407967230155; E-mail: ciwright26@hotmail.com

Key words: syncope, diet, blood pressure

Received: October 23, 2016; Accepted: November 08, 2016; Published: November 11, 2016 


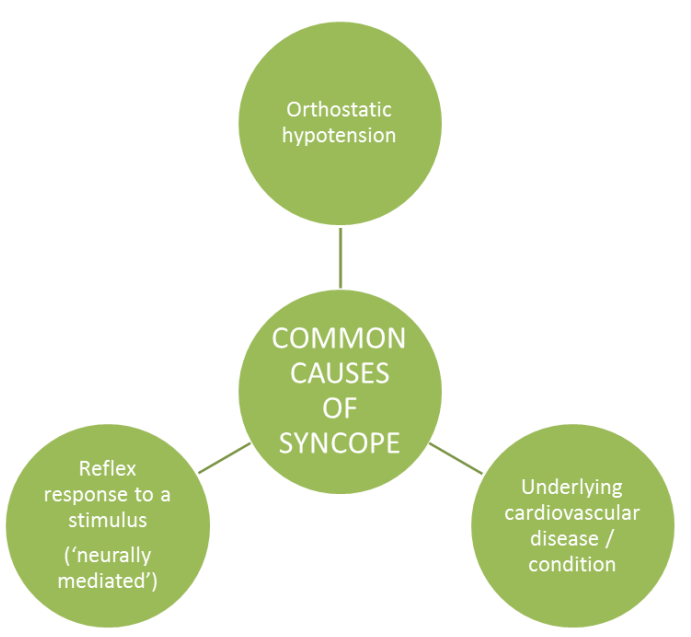

Figure 1. Three common causes of syncope [1].

\section{Dietary advice and syncope}

In January 2012, the Syncope Trust and Reflex Anoxic Seizures (STARS) [11] published an article entitled 'You are what you eat-a guide to healthy eating for patients with vasovagal syncope and postural tachycardia syndrome (PoTS)'. This information was developed to help patients with PoTS to better manage their fluid and food intake, and to help to avoid and alleviate the symptoms of syncope.

In terms of fluid intake, it is advised that patients imbibe water prior to and following exercise. The same advice is also offered on hot days along with avoidance of drinking alcoholic beverages. Moreover, patients were advised to consider drinking sports drinks to rehydrate and to increase the intake of electrolytes and glucose. And, to even consider caffeine drinks such as coffee, tea and coca cola drinks. The explanation was that caffeine leads to a small and acute rise in blood pressure ('pressor effect'), which counteracts the small decreases in arterial pressure seen in syncope patients. Drinking two glasses of water has a similar pressor effect and is thought to be explained by the stimulation of stretch nerve fibres in the stomach.

Dietary advice includes a number of food stuffs, including low gastro-intestinal (GI) and high fibre diets, as well as salts such as potassium. Indeed, the American Heart Association, recommends the intake of salt in patients with neurally mediated syncope (also known as neurocardiogenic, vasovagal, vasodepressor or reflex mediated syncope), along with the advice to drink plenty of water to avoid dehydration and a drop in arterial blood pressure.

Foods with a low GI release sugar as a slower rate and this reduces plasma peak levels as well as prolonging the delivery of sugar. Examples include bran or oat breakfast cereal, nuts, yoghurt, beans, fruit and vegetables, brown rice and pasta and wholegrain bread. Eating food stuffs with a low GI index diets are advised in patients with PoTS. Indeed, the UK Department of Health has endorsed materials published by STARS [11] where it is stated that: "Many patients with PoTS experience deterioration in their symptoms after meals. This is because blood is diverted to the splanchnic blood vessels which supply the organs in the abdomen in an effort to digest food. Meals rich in refined carbohydrate (e.g. sugars and white flour) worsen this problem".

Fibre-rich diets, along with increased fluid intake, are also advised in patients with syncope to help to avoid constipation which can be exacerbated in patients with syncope. Diets should include foods such as bran cereal, fruits (both fresh and dried), vegetables (including beans and lentils) as well as wholemeal foods like bread, rice and even pasta. Lastly, patients taking medicines to manage their symptoms should also consider taking additional supplements. For example, fainting events have been reported in patients with PoTS and syncope that have taken fludrocortisone to manage their condition [12]. The explanation being based on a decline in potassium levels and therefore the intake of potassium-rich foods is recommended and include bananas, avocados, dates, beans and lentils, spinach, mushrooms, melon and dried fruits.

\section{Conclusions}

Syncope is reported to affect around one in three people and at some point, everyone will experience similar symptoms, which are commonly experienced when standing up too quickly after taking a warm bath. Syncope occurs after a momentary loss of consciousness (experienced as dizziness) associated with a temporary, insufficient supply of oxygenated blood to the brain. Other short-term effects include a loss of muscle strength, a feel of nausea and sweaty hands. Syncope occurs when arterial blood pressure is depressed (i.e., orthostatic hypotension) or drops in response to a reflex (i.e., fear of needles or blood) or due to an underlying cardiovascular disease or condition. Diet is one approach to managing the symptoms of syncope and includes advice on the intake of fluids and foods. Caffeine, water and sports/electrolyte enriched drinks can be helpful to counter reduced arterial pressure and rehydrate. Patients with syncope should consider their diet to manage their symptoms. Several foods have been covered in this article and include diets that have a high fibre and/salt content as well as foods with a low GI carbohydrate index.

\section{Acknowledgements}

The author would like to acknowledge the writing support provided by Red Pharm communications, which is part of the Red Pharm company (please see @RedPharmCo on Twitter).

\section{References}

1. Kidd SK, Doughty C, Goldhaber S (2016) Syncope (Fainting). Circulation133 e600-e602.

2. Sheldon RS (2016) Fainting research.

3. Sheldon RS, Wright CI, Duff HJ, Thakore E, Gillis AM, et al. (2007) Mechanism of hypotensive transients associated with abrupt bradycardias in conscious rabbits. Can J Cardiol 23: 721-726. [Crossref]

4. Coughlan T (2016) Royal guards who are taught how to faint. Express, April 222011.

5. Edholm E (1957) The mechanism of fainting. The New Scientist 1: 14-16.

6. Oberg B, Thoren P (1973) Circulatory responses to stimulation of left ventricular receptors in the cat. Acta Physiologica 88: 8-22.

7. Wright C, Drinkhill MJ, Hainsworth R (2000) Reflex effects of independent stimulation of coronary and left ventricular mechanoreceptors in anaesthetised dogs. J Physiol528: 349-358.[Crossref]

8. BBC Sports webpage (2016) Foot ball.

9. American Heart Association webpage (2016) Definition of Hypertrophic Cardiomyopathy.

10. Spirito P (2009) Syncope and Risk of Sudden Death in Hypertrophic Cardiomyopathy. Circulation 119: 1703-1710. [Crossref]

11. Department of Health (2012) You are what you eat-a guide to healthy eating for patients with vasovagal syncope and PoTS 2012.

12. Medicines.org.uk (2016) Summary of Product Characteristics for Fludrocortisone acetate $0.1 \mathrm{mg}$ tablets.

Copyright: (C)2016 Wright CI. This is an open-access article distributed under the terms of the Creative Commons Attribution License, which permits unrestricted use, distribution, and reproduction in any medium, provided the original author and source are credited. 